\title{
A New Glucocerebrosidase Chaperone Reduces $\alpha$-Synuclein and Glycolipid Levels in iPSC-Derived Dopaminergic Neurons from Patients with Gaucher Disease and Parkinsonism
}

\author{
Elma Aflaki, ${ }^{1}$ Daniel K. Borger, ${ }^{1}$ Nima Moaven, ${ }^{1}$ B Barbara K. Stubblefield, ${ }^{1}$ Steven A. Rogers, ${ }^{2}$ Samarjit Patnaik, ${ }^{3}$ \\ Frank J. Schoenen, ${ }^{2}$ Wendy Westbroek, ${ }^{1}$ CWei Zheng, ${ }^{3}$ Patricia Sullivan, ${ }^{4}$ Hideji Fujiwara, ${ }^{5}$ Rohini Sidhu, ${ }^{5}$

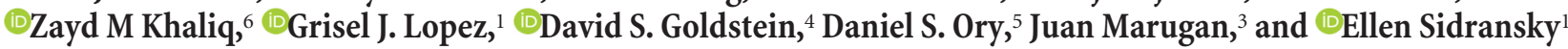 \\ ${ }^{1}$ Section of Molecular Neurogenetics, National Human Genome Research Institute, ${ }^{3}$ National Center for Advancing Translational Sciences, ${ }^{4} \mathrm{Clinical}$ \\ Neurocardiology Section, and ${ }^{6}$ Cellular Neurophysiology Unit, National Institute of Neurological Disease and Stroke, National Institutes of Health, \\ Bethesda, Maryland 20892, ${ }^{2}$ University of Kansas Specialized Chemistry Center, University of Kansas, Lawrence, Kansas 66047, and ${ }^{5}$ Diabetic \\ Cardiovascular Disease Center and Department of Medicine, Washington University School of Medicine, St. Louis, Missouri 63110
}

Among the known genetic risk factors for Parkinson disease, mutations in GBA1, the gene responsible for the lysosomal disorder Gaucher disease, are the most common. This genetic link has directed attention to the role of the lysosome in the pathogenesis of parkinsonism. To study how glucocerebrosidase impacts parkinsonism and to evaluate new therapeutics, we generated induced human pluripotent stem cells from four patients with Type 1 (non-neuronopathic) Gaucher disease, two with and two without parkinsonism, and one patient with Type 2 (acute neuronopathic) Gaucher disease, and differentiated them into macrophages and dopaminergic neurons. These cells exhibited decreased glucocerebrosidase activity and stored the glycolipid substrates glucosylceramide and glucosylsphingosine, demonstrating their similarity to patients with Gaucher disease. Dopaminergic neurons from patients with Type 2 and Type 1 Gaucher disease with parkinsonism had reduced dopamine storage and dopamine transporter reuptake. Levels of $\alpha$-synuclein, a protein present as aggregates in Parkinson disease and related synucleinopathies, were selectively elevated in neurons from the patients with parkinsonism or Type 2 Gaucher disease. The cells were then treated with NCGC607, a small-molecule noninhibitory chaperone of glucocerebrosidase identified by high-throughput screening and medicinal chemistry structure optimization. This compound successfully chaperoned the mutant enzyme, restored glucocerebrosidase activity and protein levels, and reduced glycolipid storage in both iPSC-derived macrophages and dopaminergic neurons, indicating its potential for treating neuronopathic Gaucher disease. In addition, NCGC607 reduced $\alpha$-synuclein levels in dopaminergic neurons from the patients with parkinsonism, suggesting that noninhibitory small-molecule chaperones of glucocerebrosidase may prove useful for the treatment of Parkinson disease.

Key words: $\alpha$-synuclein; dopaminergic neurons; glucocerebrosidase; induced pluripotent stem cells; parkinsonism; pharmacological chaperone

Significance Statement

Because GBA1 mutations are the most common genetic risk factor for Parkinson disease, dopaminergic neurons were generated from iPSC lines derived from patients with Gaucher disease with and without parkinsonism. These cells exhibit deficient enzymatic activity, reduced lysosomal glucocerebrosidase levels, and storage of glucosylceramide and glucosylsphingosine. Lines generated from the patients with parkinsonism demonstrated elevated levels of $\alpha$-synuclein. To reverse the observed phenotype, the neurons were treated with a novel noninhibitory glucocerebrosidase chaperone, which successfully restored glucocerebrosidase activity and protein levels and reduced glycolipid storage. In addition, the small-molecule chaperone reduced $\alpha$-synuclein levels in dopaminergic neurons, indicating that chaperoning glucocerebrosidase to the lysosome may provide a novel therapeutic strategy for both Parkinson disease and neuronopathic forms of Gaucher disease. 


\section{Introduction}

Mutations in GBA1, the gene mutated in Gaucher disease (GD), are the most frequent known genetic risk factor for the common neurodegenerative disorders Parkinson disease (PD) and dementia with Lewy bodies (DLBs). GD is caused by the inherited deficiency of the enzyme glucocerebrosidase (GCase, E.C.3.2.1.45), which hydrolyzes the glycolipids glucosylceramide (GlcCer) and glucosylsphingosine (GlcSph), and is characterized by lysosomal storage of these substrates. GD is classified into three types based on the absence (Type 1) or rate of progression of neurologic manifestations (Types 2 and 3). Type 2 GD is a progressive and lethal disorder of infancy or early childhood (Weiss et al., 2015).

Studies of cohorts from around the world demonstrate that patients with PD and DLB are far more likely to carry mutations in GBA1 than controls (Sidransky et al., 2009; Nalls et al., 2013). Furthermore, patients with GD and their carrier relatives have an increased risk of both PD and DLB (Tayebi et al., 2003; GokerAlpan et al., 2004).

While the appreciation of this association has directed attention to the role of the lysosome in these neurodegenerative disorders, the pathological mechanisms by which mutations in GBA1 contribute to parkinsonism remain unclear. Theories proposed implicate enhanced levels of $\alpha$-synuclein ( $\alpha$-syn), a protein with a propensity to aggregate, as well as sphingolipid accumulation and impaired intracellular trafficking, all of which may play a role in the pathogenesis of neurodegeneration (Mazzulli et al., 2011; Siebert et al., 2014). There is increasing evidence of an inverse relationship between levels of GCase and $\alpha$-syn in the brain, as GCase levels and activity are diminished even in idiopathic PD (Gegg et al., 2012; Murphy et al., 2014). Clinically, GBA1-associated parkinsonism in homozygotes or carriers often mirrors sporadic $\mathrm{PD}$ with regard to the presence of tremor, bradykinesia, and rigidity (Schapira, 2015) as well as in imaging studies. However, some patients manifest clinical and imaging features and pathology consistent with DLB (Kono et al., 2010; Saunders-Pullman et al., 2010; Goker-Alpan et al., 2012). Both PD and DLB are synucleinopathies, characterized by the accumulation of LBs containing aggregated $\alpha$-syn, albeit in different anatomic distributions. Thus, a major therapeutic goal is to identify strategies to prevent aggregation or to facilitate the clearance of aggregated proteins.

The inverse relationship between GCase and $\alpha$-syn led us to posit that enhancement of GCase activity, a therapeutic strategy under development for GD, could also have implications for the treatment of PD. While many symptoms of GD are effectively treated with enzyme replacement therapy or substrate reduction therapy, these costly therapies are ineffective for neuronopathic forms of GD (Altarescu et al., 2001). Pharmacological chaperones have been proposed as an alternative therapy for GD, including neuronopathic forms, and potentially for PD (Sawkar et al., 2006;

Received Feb. 25, 2016; revised April 28, 2016; accepted May 25, 2016.

Author contributions: E.A., F.J.S., W.Z., J.M., and E.S. designed research; E.A., D.K.B., N.M., B.K.S., S.A.R., S.P., F.J.S., W.W., P.S., H.F., R.S., Z.M.K., G.J.L., and J.M. performed research; E.A., B.K.S., S.A.R., F.J.S., W.W., W.Z., Z.M.K., D.S.G., D.S.O., and J.M. contributed unpublished reagents/analytic tools; E.A., D.K.B., N.M., S.A.R., S.P., F.J.S., H.F., D.S.G., D.S.O., J.M., and E.S. analyzed data; E.A. and E.S. wrote the paper.

This work was supported by the Intramural Research Programs of the National Human Genome Research Institute, National Center for Advancing Translational Sciences, National Institute of Neurological Disorders and Stroke, and National Institutes of Health and in part by National Human Genome Research Institute Grant U54HG005031.

The authors declare no competing financial interests.

Correspondence should be addressed to Dr. Ellen Sidransky, Section on Molecular Neurogenetics, Medical Genetics Branch, National Human Genome Research Institute, National Institutes of Health, Building 35, Room 1E623, 35 Convent Drive, MSC 3708, Bethesda, MD 20892-3708. E-mail: sidranse@mail.nih.gov.

DOI:10.1523/JNEUROSCI.0636-16.2016

Copyright $\odot 2016$ the authors $\quad 0270-6474 / 16 / 367442-12 \$ 15.00 / 0$
Table 1. Genotypes of patients with Gaucher disease used for iPSC lines

\begin{tabular}{lllll}
\hline & Genotype & & Gender & Age \\
\hline GD1-1 & N370S/N370S & Sib1 & Male & $64 \mathrm{yr}$ \\
GD1-PD1 & N370S/N370S & Sib2 & Male & $62 \mathrm{yr}$ \\
GD1-2 & N370S/N370S & & Male & $66 \mathrm{yr}$ \\
GD1-PD2 & N370S/C0.84dupG & & Male & $59 \mathrm{yr}$ \\
GD2 & IVS2+1G $>$ T/L444P & & Female & $9 \mathrm{mo}$ \\
\hline
\end{tabular}

Richter et al., 2014). Such drugs are designed to bind to mutant GCase and facilitate its refolding and subsequent trafficking to the lysosome. Using high-throughput screening, we identified two series of compounds, a thiazide series and a new salicylic acid series, which successfully translocate GCase to the lysosome and can cross into the brain (Goldin et al., 2012; Aflaki et al., 2014). However, it has been difficult to evaluate the effects of such drugs, especially in neurons, due to the scarcity of appropriate cellular models.

Induced pluripotent stem cells (iPSCs) offer an opportunity to study functional dopaminergic neurons derived from patients with GD and PD. In this study, we differentiated iPSCs from patients with non-neuronopathic GD (GD1), GD with parkinsonism (GD1-PD), and acute neuronopathic GD (GD2) into dopaminergic (iDA) neurons and macrophages (iMacs). These new models have enabled us to evaluate GCase activity and protein levels, intracellular dopamine (DA) content and metabolism, and $\alpha$-syn levels. These cells were also treated with a new noninhibitory GCase chaperone, enabling us to assess the ability of this compound to correct the cellular phenotype. Utilizing the iMacs and iDA neurons derived from our patients, we demonstrate how targeting glucocerebrosidase activity in these models provides insight into disease pathogenesis, identifying new avenues for the development and evaluation of drugs for GD and potentially PD.

\section{Materials and Methods}

Synthesis of NCGC607. Synthetic reactions (see Fig. 1) were performed under an atmosphere of dry argon or nitrogen. All anhydrous solvents, commercially available starting materials, and reagents were purchased from Sigma-Aldrich. Analytical thin-layer chromatography (TLC) was performed using Sigma-Aldrich thin-layer chromatography plates $(5 \times$ $20 \mathrm{~cm}, 60 \AA, 250 \mu \mathrm{m})$. Visualization was accomplished by irradiation under a $254 \mathrm{~nm}$ UV lamp. Reverse-phase preparative purification was performed on a Waters semipreparative high-performance liquid chromatography (HPLC) system (Column: Phenomenex Luna C18 $-5 \mu \mathrm{m}$, $30 \times 75 \mathrm{~mm})$ at a flow rate of $45 \mathrm{ml} / \mathrm{min}$; mobile phase consisted of acetonitrile and water (each containing $0.1 \%$ trifluoroacetic acid). A gradient of $10 \%-50 \%$ acetonitrile over 8 min was used during the purification. Fraction collection was triggered by UV detection $(220 \mathrm{~nm}) .{ }^{1} \mathrm{H}$ spectra were recorded on a Varian Inova $400 \mathrm{MHz}$ spectrometer. Chemical shifts are reported in ppm with the solvent resonance as the internal standard (DMSO- $d_{6} 2.50 \mathrm{ppm}$, for $\left.{ }^{1} \mathrm{H}\right)$. Data are reported as follows: chemical shift, multiplicity $(\mathrm{s}=$ singlet, $\mathrm{d}=$ doublet, $\mathrm{t}=$ triplet, $\mathrm{q}=$ quartet, br $\mathrm{s}=$ broad singlet, $\mathrm{m}=$ multiplet), coupling constants, and number of protons. Molecular weights were confirmed using an Agilent Time-Of-Flight Mass Spectrometer (Agilent Technologies). A 3 min gradient from $4 \%$ to $100 \%$ acetonitrile $(0.1 \%$ formic acid) in water was used with a $4 \mathrm{~min}$ run time at a flow rate of $1 \mathrm{ml} / \mathrm{min}$. A Zorbax SB-C18 column $(3.5 \mu \mathrm{m}, 2.1 \times 30 \mathrm{~mm})$ was used at $50^{\circ} \mathrm{C}$. Molecular formula were confirmed using electrospray ionization in the positive mode with the Agilent Masshunter software (Agilent Technologies, version B.02).

Patient samples. Six iPSC lines were derived from patients with GD1 and GD2 with different mutations and from an adult control (Table 1) as described previously (Aflaki et al., 2014). All were generated from fibroblasts taken from individuals evaluated clinically for up to a decade by a Movement Disorder specialist at the Clinical Center of the National In- 
stitutes of Health. Participants provided informed consent under a National Human Genome Research Institute Institutional Review Boardapproved protocol. Two of the patients with GD1 also developed PD. Patient GD1-PD1 with genotype N370S/N370S was diagnosed with PD at the age of 52. His brother (GD1-1), also with genotype N370S/N370S, and now 64 years old, has GD but no manifestations of PD. Patient GD1-PD2 with genotype N370S/c.84dupG developed parkinsonian manifestations at age 45 and died at age 59 with a postmortem diagnosis of DLB.

Differentiation of iPSCs to monocytes and macrophages. Differentiation of iPSCs to monocytes and the macrophages, performed to establish compound efficacy, was described previously (Aflaki et al., 2014). In summary, embryoid bodies (EBs), formed using AggreWell 400 plates (Stem Cell Technology), were transferred to ultra-low adherence plates in AggreWell medium supplemented with $10 \mu \mathrm{M}$ ROCK inhibitor (Stem Cell Technology) for $4 \mathrm{~d}$, with daily media changes. For differentiation to monocytes, EBs were transferred to gelatin (1\%)-coated 6 well plates, in Advanced DMEM (Invitrogen) supplemented with $10 \%$ FCS, $0.055 \mathrm{~mm} \beta$-mercaptoethanol, $50 \mathrm{ng} / \mathrm{ml}$ $\mathrm{M}-\mathrm{CSF}$, and $25 \mathrm{ng} / \mathrm{ml} \mathrm{IL}-3$ (R\&D Systems). Media was replaced every $3 \mathrm{~d}$. Monocytes were harvested from the supernatant, centrifuged at $1500 \mathrm{rpm}$ for $5 \mathrm{~min}$, and differentiated into iMacs using RPMI supplemented with $10 \%$ FCS and $100 \mathrm{ng} / \mathrm{ml} \mathrm{M-CSF}$.

Differentiation of iPSC to dopaminergic neurons. iPSCs were detached and cultured in AggreWell 800 (Stem Cell Technology) for $5 \mathrm{~d}$. The resulting EBs were seeded into plates coated with polyornithine/laminin for $6 \mathrm{~d}$. At day 11, typical neural tube-like rosettes appeared and were detached using STEMdiff Neural Rosette Selection (Stem Cell Technology). Rosettes were cultured into poly-ornithine/laminin-coated plates and differentiated to neuronal progenitor cells (NPCs). Then, differentiation to dopaminergic neurons was performed based on the protocol of Hartfield et al. (2014). All growth factors were obtained from Peprotech, unless otherwise stated. Briefly, neuronal induction medium I (DMEM/ F12 supplemented with L-glutamine [2 mM], N2 supplement, BSA [1 $\mathrm{mg} / \mathrm{ml}$ ], Y27632 [10 $\mu \mathrm{M}$; Tocris Bioscience], SB431542 [10 $\mu \mathrm{M}$, Tocris Bioscience $]$ and noggin $[200 \mathrm{ng} / \mathrm{ml}]$ ) was added to the NPCs for $3 \mathrm{~d}$ and then media was changed at day 4 by withdrawing SB43152 and noggin and adding $200 \mathrm{ng} / \mathrm{ml}$ sonic hedgehog [SHH C24II], fibroblast growth factor-8a (FGF8a, $100 \mathrm{ng} / \mathrm{ml}$ ), and ascorbic acid (200 $\mu \mathrm{M}$; Sigma) for $10 \mathrm{~d}$. Cells could then be passaged and were maintained in their final differentiation media (DMEM/F12 supplemented with L-glutamine [2 $\mathrm{mm}$ ], N2 supplement, BDNF [20 $\mathrm{g} / \mathrm{ml}$ ], and glial-derived neurotrophic factor [GDNF, $20 \mu \mathrm{g} / \mathrm{ml}]$ ). For most experiments, cells were kept in culture for $>100 \mathrm{~d}$.

Immunofluorescence staining. iDA were plated on glass chamber slides coated with poly-ornithine/laminin and iMacs, fed with erythrocyte ghosts in the presence and absence of NCGC607, were plated on glass chamber slides and fixed with 4\% PFA. Cells were blocked in PBS containing $0.1 \%$ saponin, $100 \mu \mathrm{M}$ glycine, and $2 \%$ donkey serum, followed by incubation with the primary antibody. They were washed and incubated with donkey anti-mouse or anti-rabbit secondary antibodies and conjugated to Alexa-488 or Alexa-555 (Invitrogen). Cells were mounted with Vectashield plus DAPI (Vector Laboratories) and $Z$-stack images were acquired with a Zeiss 510 META laser scanning microscope (Carl Zeiss) using a 488 argon, a $543 \mathrm{HeNe}$, and a UV laser. Images were acquired using $63 \times$ a PlanNeoFluar lens.

Immunoblotting. iDA neurons were harvested and sonicated at $4{ }^{\circ} \mathrm{C}$ in RIPA buffer $(50 \mathrm{~mm}$ Tris-HCl, pH 7.4, $150 \mathrm{~mm} \mathrm{NaCl}, 0.5 \% \mathrm{Na}$ deoxycholate, $0.1 \%$ SDS, and protease inhibitor). After quantification with BCA (Thermo Scientific), $10 \mu \mathrm{g}$ of the lysates was separated by Novex NuPAGE SDS-PAGE Gel System (Invitrogen) and transferred to iBlot PVDF membranes (Invitrogen). Blots were blocked in 1:1 PBS, Odyssey Blocking Buffer (Li-COR Bioscience) for $1 \mathrm{~h}$ at room temperature. The membrane was incubated in blocking buffer containing $0.1 \%$ Tween 20 (Sigma), and respective primary antibodies: MAO-A (105391-AP) and MAO-B (12602-1-AP) (Abcam), $\alpha$-syn (Santa Cruz Biotechnology, sc-7011-R) and Tuj1 (Cell Signaling Technology, 5568), overnight at $4{ }^{\circ} \mathrm{C}$, followed by three $15 \mathrm{~min}$ washes, and was incubated in blocking buffer containing 0.1\% Tween 20 (Sigma), 0.01\% SDS, and
IRDye 680RD secondary antibody 1:10,000 (Li-COR Bioscience) for $1 \mathrm{~h}$ at room temperature. The blot was imaged using an Odyssey imaging system (Li-CORE Bioscience) and quantified using Image Studio Lite software.

GlcCer and GalCer analysis by mass spectrometry. An aliquot of iMac or iDA cell pellet, homogenized in water, was spiked with internal standards (C17 S1P, N,N-dimethylpsychosine, GalCer(d18:1 8:0)) and extracted with methanol. One portion of the extract was used for quantification of GlcSph, and GlcCer using reverse-phase C18 columns for HPLC/MS/ MS. The other portion of the extract was used for the determination of GlcSph and GalSph as well as GlcCer and galactosylceramides (GalCer) using an HILIC column.

Measurement of catecholamine metabolites by HPLC. iDAs were collected in $400 \mu \mathrm{l}$ 20:80 (40 $\mathrm{mM} \mathrm{H}_{2} \mathrm{PO}_{4}: 200 \mathrm{~mm}$ acetic acid) and disrupted by freezing and thawing. The catechols were extracted from $200 \mu \mathrm{l}$ by alumina adsorption and were detected and quantified by HPLC with electrochemical detection as described previously (Goldstein et al., 2012).

Evaluation of DA uptake. Dopamine update in iDA neurons was measured using a neurotransmitter transporter uptake assay kit (Molecular Devices). This kit uses fluorescent substrate that mimics the biogenic amine transmitters resulting in increased intracellular fluorescence intensity. Cells were cultured in clear-bottom black plates and were treated with 10 or $20 \mu \mathrm{M}$ GBR1209 (Tocris Bioscience) for $10 \mathrm{~min}$ in HBSS buffer supplemented with $0.1 \%$ BSA to allow binding of the compound to the transporter. Dye solution was added to the cells and fluorescence (Ex/ Em, 440/520) was measured kinetically for $30 \mathrm{~min}$ at $37^{\circ} \mathrm{C}$.

GCase activity and protein levels. iDA neurons were lysed in citratephosphate extraction buffer. To exclude activity from other nonlysosomal GCases, lysates were incubated with or without $100 \mu \mathrm{M} \mathrm{CBE}$, an inhibitor specific for $G B A 1$, for $30 \mathrm{~min}$ and the amount of uninhibited activity was subtracted from the total. Assay buffer ( 1 M 4-MU $\beta$-glu to a final concentration of $10 \mathrm{~mm}$ in citrate-phosphate buffer) was added for $90 \mathrm{~min}$ at $37^{\circ} \mathrm{C}$. The reaction was halted using a stop solution $(1 \mathrm{M} \mathrm{NaOH}$ and $1 \mathrm{M}$ glycine) and fluorescence measured. To measure GCase protein levels, lysates were incubated with $100 \mathrm{~nm}$ green fluorescent MDW933, an irreversible inhibitor (Witte et al., 2010), for $90 \mathrm{~min}$ at $37^{\circ} \mathrm{C}$. After a brief centrifugation, lysates were subjected to SDS-PAGE electrophoresis. Wet gels were scanned using a Typhoon Variable Mode Imager $\left(\lambda_{\mathrm{ex}}\right.$ $488 \mathrm{~nm}$ and $\lambda_{\mathrm{em}} 520 \mathrm{~nm}$ ).

Electrophysiology. Cultured cells were placed in a heated recording chamber $\left(31^{\circ} \mathrm{C}-33^{\circ} \mathrm{C}\right)$ and perfused with ACSF containing $125 \mathrm{mM} \mathrm{NaCl}$, $25 \mathrm{~mm} \mathrm{NaHCO}_{3}, 1.25 \mathrm{~mm} \mathrm{NaH}_{2} \mathrm{PO}_{4}, 3.5 \mathrm{~mm} \mathrm{KCl}, 1 \mathrm{~mm} \mathrm{MgCl}, 2 \mathrm{~mm}$ $\mathrm{CaCl}_{2}$, and $10 \mathrm{~mm}$ glucose bubbled with $95 / 5 \% \mathrm{O}_{2} / \mathrm{CO}_{2}$. Current-clamp and voltage-clamp recordings were made with a Multiclamp 700B amplifier and digitized with a Digidata 1440A (Molecular Devices). Lowresistance patch electrodes $(3-5 \mathrm{M})$ were pulled from borosilicate glass. In voltage-clamp recordings, pipette series resistance was compensated by $65 \%-75 \%$. Internal recording solution contained the following: $122 \mathrm{~mm}$ K-methanesulfonate, $9 \mathrm{~mm} \mathrm{NaCl}, 1.8 \mathrm{~mm} \mathrm{MgCl}_{2}$, 4 mM Mg-ATP, $0.3 \mathrm{~mm}$ Na-GTP, $14 \mathrm{~mm}$ phosphocreatine, and $10 \mathrm{~mm}$ HEPES adjusted to $\mathrm{pH}$ 7.35 with $\mathrm{NaOH}$.

RNA isolation and real-time PCR. A total of $1 \mu \mathrm{g}$ total RNA, isolated from macrophages using the PrepEase RNA/Protein Spinkit (Affymetrix 78767), was reverse transcribed using the High Capacity cDNA Reverse Transcription kit (Applied Biosystems, 4368814). Quantitative real-time PCR was performed using an ABI prism 7900 real-time PCR instrument (Applied Biosystems). Expression of $\alpha$-syn was performed using TaqMan (Applied Biosystems) with HPRT as an internal control. Two independent experiments were performed.

Statistical analysis. Statistical analyses were performed using GraphPadPrism5.0 software. Significance was determined by a Student's $t$ test using a Wilcoxon signed rank test. Correlations were determined using Pearson correlations. Data from two groups or $>2$ independent variables were analyzed by ANOVA, followed by the Bonferroni post hoc test. Data are presented as mean $\pm \mathrm{SD}$ values. 


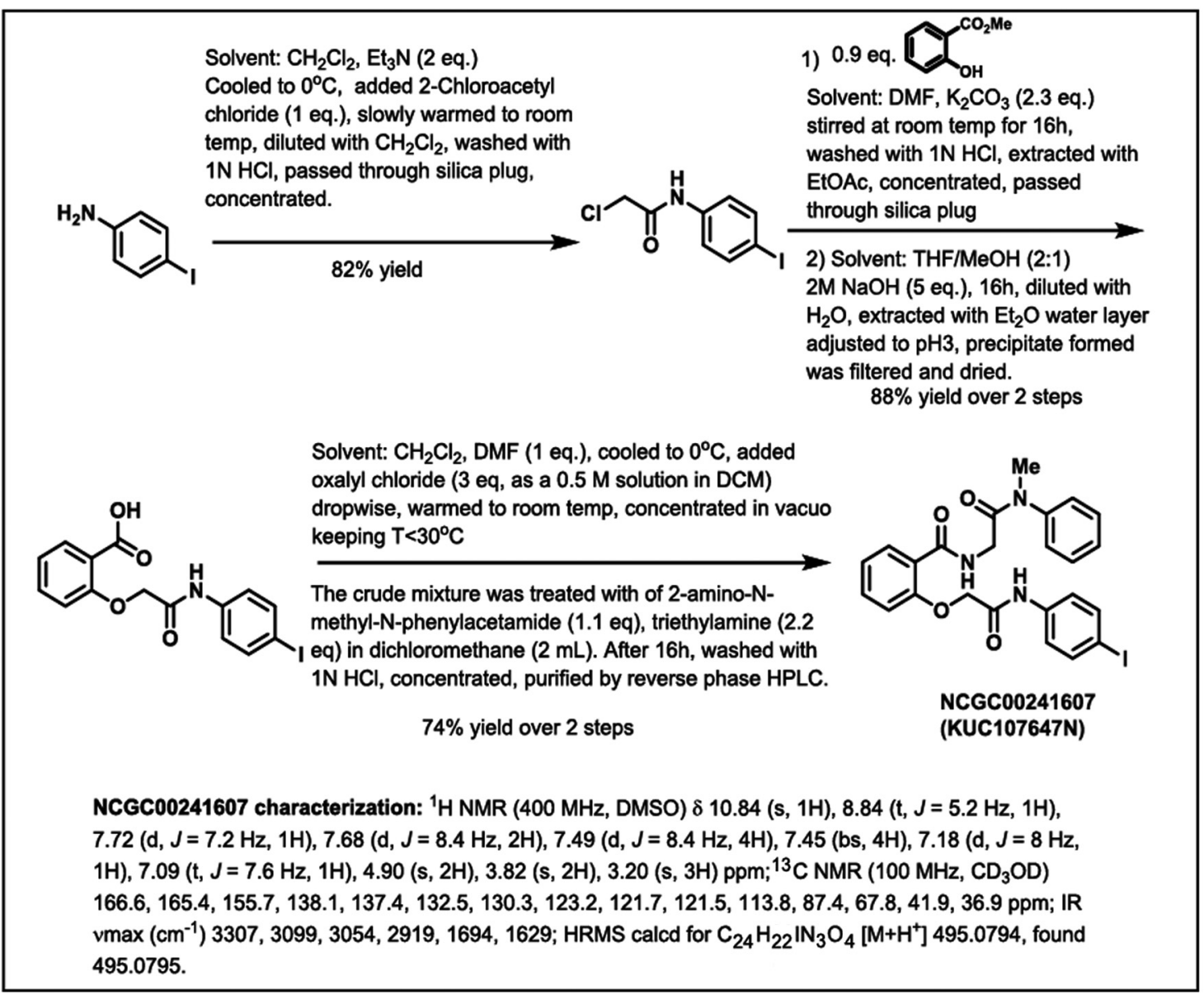

Figure 1. Synthesis of NCGC00241607. The synthesis of NCGC00241607 (NCGC607) was executed according to the scheme above. A synopsis of the procedure used in each transformation is given along with the synthetic yield and ${ }^{1} \mathrm{H}$ NMR, ${ }^{13} \mathrm{C}$ NMR, and HRMS data characterizing NCGC607.

\section{Results}

NCGC607, a new noninhibitory chaperone, increases GCase and reduces GlcCer levels in Gaucher macrophages

NCGC607, a salicylic acid derivative, is a noninhibitory smallmolecule chaperone that belongs to a chemical series first identified by high throughput screening using a patient spleen sample as the source of mutant human GCase (Goldin et al., 2012). The specific compound NCGC607 was discovered using medicinal chemistry to improve the activity of mutant GCase in the screening assay (Fig. 1).

The cellular phenotype of GD primarily manifests in macrophages, where excessive glucosylceramide (GlcCer) accumulates in lysosomes due to defective digestion of erythrocyte or white blood cell membranes. Therefore, to establish the ability of this new compound to chaperone GCase, we first evaluated the effect of NCGC607 using iPSC-derived patient macrophages (iMacs), produced as described previously (Aflaki et al., 2014). In iMacs derived from patients with genotype N370S/N370S (GD1) and IVS2 + 1G>T/L444P (GD2), treatment with $3 \mu \mathrm{M}$ NCGC607 significantly enhanced GCase activity and increased GCase levels (Fig. $2 A, B$ ). In the presence of NCGC607, GD iMacs showed decreased levels of GlcCer (Fig. 2C) and enhanced translocation of GCase to the lysosome (Fig. 2D,E). Together, these data indicate that NCGC607 effectively chaperoned GCase, increasing translocation, enhancing enzyme activity, and reducing GlcCer levels.
Gaucher iPSC-dopaminergic neurons appropriately recapitulate the human phenotype with diminished GCase activity and levels

Next, we differentiated our six iPSC lines to iDA neurons using a modified published protocol (Kriks et al., 2011). The EBs in the colony center developed a columnar morphology, and, after $4 \mathrm{~d}$ in culture, became organized into neural-tube-like rosettes (Fig. 3A), which expressed Sox1, an early transcription factor expressed in ectodermal cells committed to a neuronal fate (Pevny et al., 1998). Rosette-containing clusters were expanded to form NPCs, which stained positive for neuronal markers FOXA2, Nestin, and neuron-specific $\beta$-III-tubulin (Tuj1). After $12 \mathrm{~d}$ of differentiation, most cells expressed PITX3, FOXA2, and AADC mRNA (Fig. 3B). The GD1 and GD1-PD NPCs showed the expected reduction in GCase activity ( $<30 \%$ of control levels), whereas the GD2 NPCs had $<2 \%$ GCase activity (Fig. $3 C$ ). All had correspondingly low levels of GCase protein (Fig. 3D). The neurons were further differentiated to iDA neurons, and immunoflorescence staining confirmed that they expressed Map2, Tuj1, and the iDA marker TH (Fig. $3 A, D, E$ ).

iPSC-derived iDA neurons appear to be biologically functional

To establish that these were indeed iDA neurons, we examined catecholamine metabolism, exploring storage, release, and uptake in cells kept in culture for $>100$ d. Figure $4 A$ shows the 
A

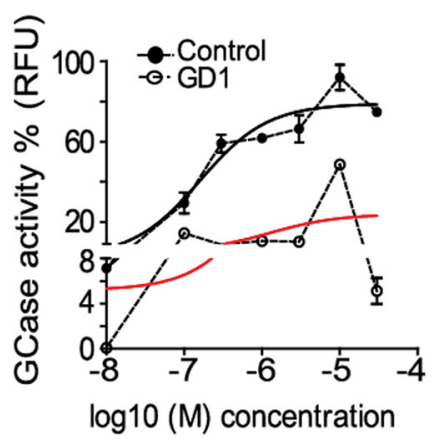

B

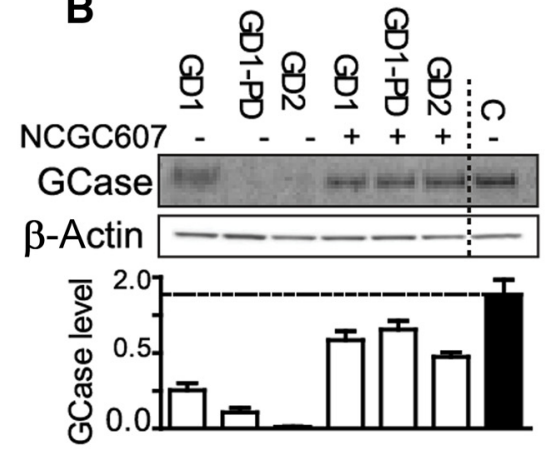

C

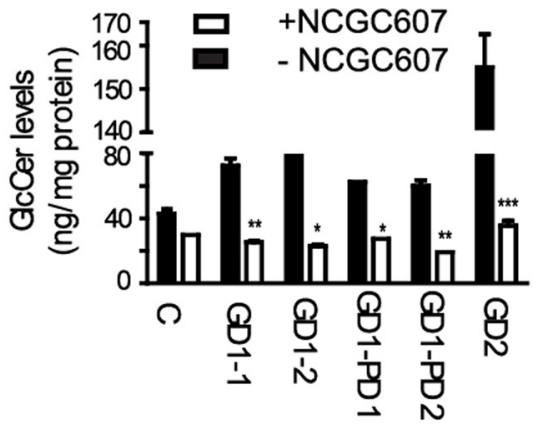

$\mathbf{E}$
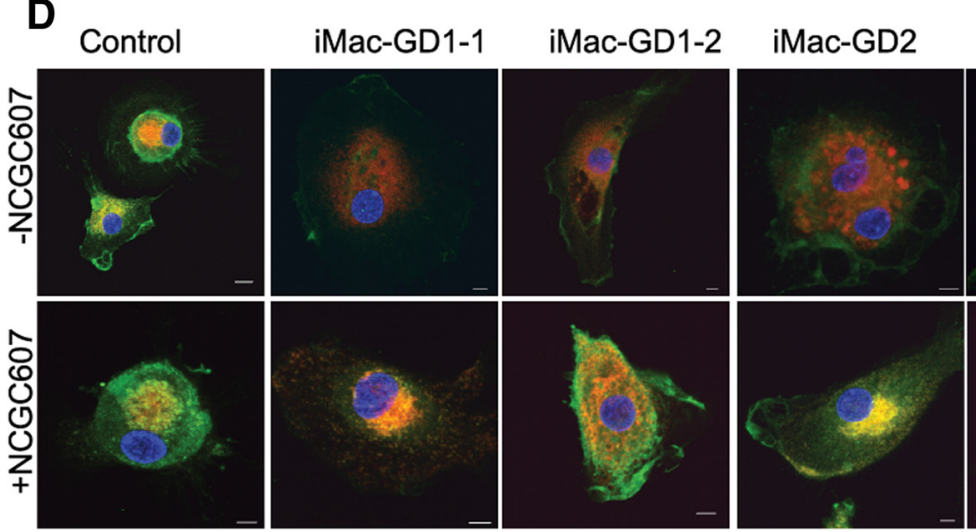

iMac-GD1-PD
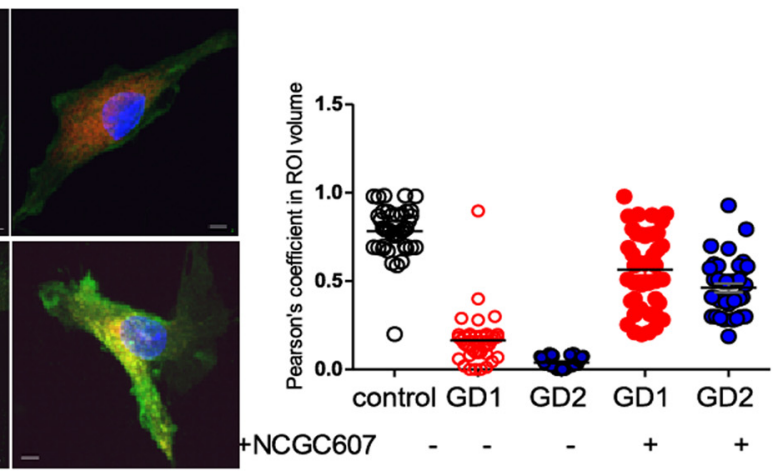

Figure 2. NGGC607 enhances GCase activity and facilitates its translocation to lysosomes in Gaucher macrophages. $A$, GCase activity in the presence of different concentrations of NCGC607 measured in iMacs from a control and iPSC line GD1-1 (N370S/N370S). Graph represents two independent experiments. RFU, Relative fluorescence units. B, Western blot analysis of GCase in iMacs from two subjects with GD1 (N370S/N370S) and one with Type 2 with and without NCGC607 (3 $\mu \mathrm{m}$ for $6 \mathrm{~d}$ ). Control iMacs (C) are shown on the right. $\beta$-Actin is used as the loading control. Quantification of the results as the ratio of GCase to $\beta$-actin appears in the graph below. C, GlCCer levels were measured using HPLC/MS/MS in control and iMacs from the iPSC lines indicated. Graph represents two independent experiments (each experiment in triplicate). Statistically significant differences between patient and control values: ${ }^{*} p=0.03 ;{ }^{* *} p=0.0092$ and $p=0.0046 ;{ }^{* * * *} p=$ 0.0005. D, Immunofluorescence study of iMacs treated with NCG607 (3 $\mu$ M) for $6 \mathrm{~d}$ and costained for GCase (green), lysosomal marker Lamp2 (red), and nuclear marker DAPI (blue). Z-stack images were acquired using a Zeiss 510 confocal microscope $(63 \times$ magnification). Scale bars, $5 \mu \mathrm{m}$. $\boldsymbol{E}$, Pearson's coefficient was quantified reflecting the degree of colocalization between $\mathrm{GCase}$ and Lamp2 using Imaris software. Each data point represents the mean value of the cells in a field.

capacity of synthesis and release of DA in iPSC-derived iDA neurons using HPLC to measure the intracellular levels of both DA and 3,4-dihydroxyphenylacetic acid. Compared with controls, there is reduced intracellular DA in GD1-PD and GD2, but not GD1, iDA neurons.

DA uptake is reduced in Gaucher iDA neurons from patients with parkinsonism

Next, DA uptake was measured in iDA neurons kept in culture for $150 \mathrm{~d}$ in the presence or absence of GBR1209, a selective inhibitor of the DA transporter (DAT). DAT, expressed in iDA neurons, pumps the neurotransmitter DA out from the synapses, returning it to the cytosol. Administering either 10 or $20 \mu \mathrm{M}$ GBR1209 for $30 \mathrm{~min}$, we observed reduced DA uptake in GD1 and GD1-PD iDA neurons. However, in GD1-PD neurons, DA uptake was lowered further with $20 \mu \mathrm{M}$ GBR1209 (Fig. 4B). While DAT1 is expressed in iDA neurons (Fig. 4C), mRNA levels of DAT1 were reduced in the GD1-PD, but not the GD2 or GD1 iDA neurons (Fig. 4D). iDA neurons also express the vesicular monoamine transporter 2 (VMAT2) (Fig. 4E), which is responsible for packaging DA into cellular compartments (Nirenberg et al., 1996). We found that VMAT2 mRNA levels were also lower in GD1-PD iDA neurons (Fig. 4F). Overall, these data indicate that DA uptake was reduced in GD1-PD iDA neurons. Electrophysiology studies also indicated that the iPSC-derived iDA neurons were functional. After $40 \mathrm{~d}$ in culture, they fired and showed appropriate action potentials in response to injected current using a patch clamp, exhibiting voltage-gated sodium and potassium currents (Fig. 4G).

Treatment of Gaucher iDA neurons with NCGC607 increased GCase activity, enhanced translocation of mutant GCase to the lysosome, and reduced substrate levels

GD1, GD1-PD, and GD2 iDA neurons were cultured for $100 \mathrm{~d}$ and then treated with the small-molecule NCGC607 for $21 \mathrm{~d}$. GCase levels, reduced in GD iDA neurons, improved after treatment with NCGC607 (Fig. 5A, B). The residual GCase activity in GD1, GD1-PD, and GD2 iDA neurons was $40 \pm 1.2 \%, 25.35 \pm$ $2.07 \%$, and $2 \pm 0.2 \%$ of control activity, respectively. In the presence of NCGC607, GCase activity increased two-fold in GD1 and 1.8-fold GD1-PD iDA neurons. In GD2 iDA neurons treated with NCGC607, GCase activity increased 40-fold (Fig. 5C).

In iDA neurons from each patient with GD, treatment with compound NCGC607 resulted in markedly increased translocation of GCase to the lysosome (Fig. 5D), as reflected by enhanced colocalization of GCase with the lysosomal marker Lamp2 (Fig. 5E).

Next, levels of GlcSph, a toxic substrate of GCase especially abundant in the brain, were measured using mass spectrometry in iDA neurons held in culture for $>150 \mathrm{~d}$. iDA neurons from the 
A

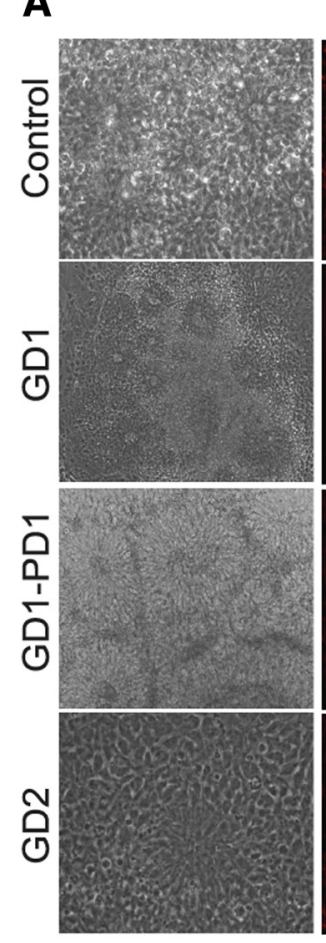

B

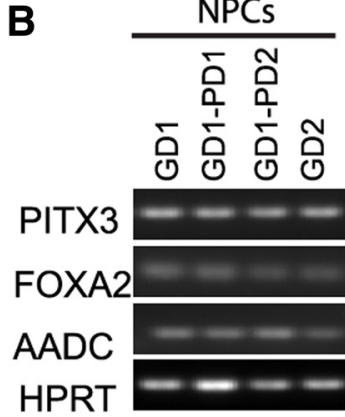

Sox1
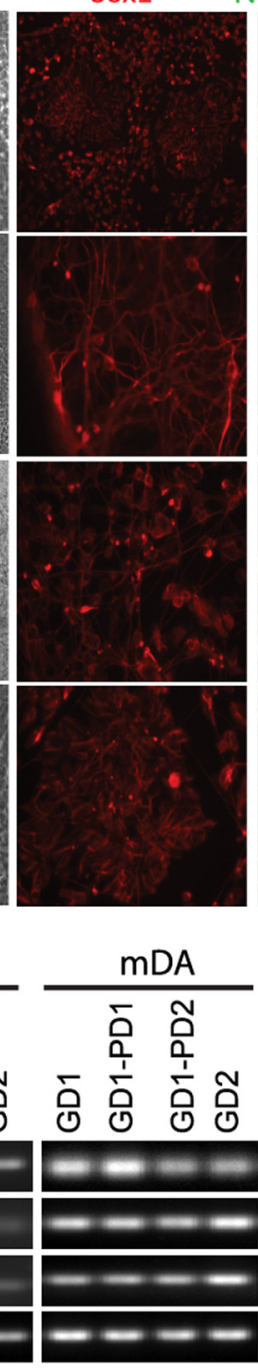

Nestin/FOXA2/Tuj1 Nestin/Map2

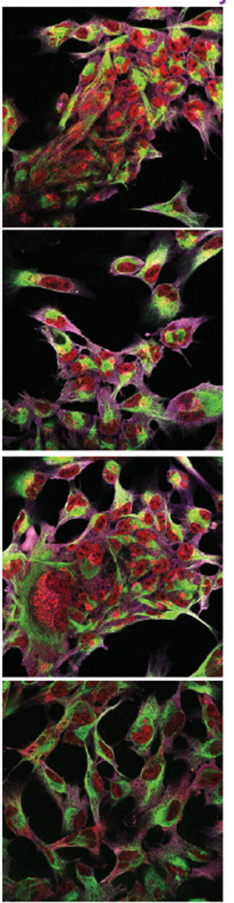

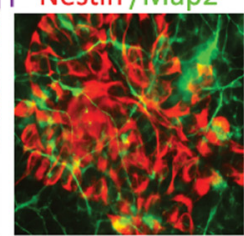
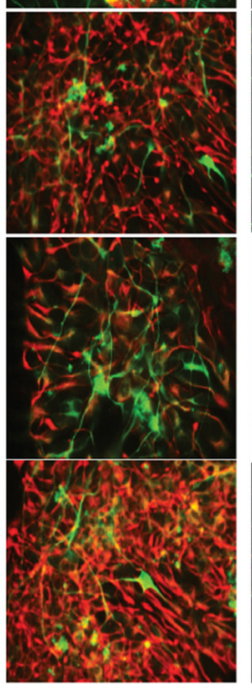

E

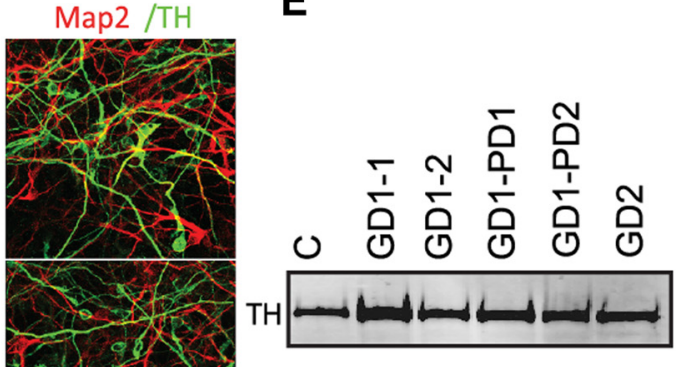

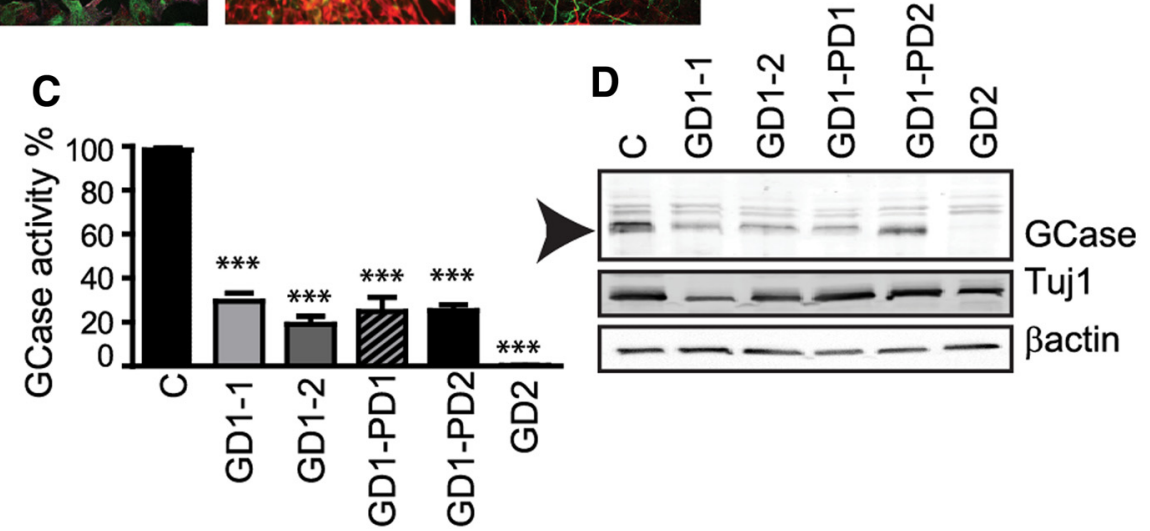

Figure 3. Differentiation of iDA neurons from GD or GD-PD iPSCS. A, Each iPSC line was differentiated into rosettes (first panel), NPCS (second panel), and then iDA neurons and analyzed by immunofluorescence staining during the different stages of differentiation. Stains include antibodies to Sox1 (red), Nestin/FoxA2/TuJ1 (green/red/pink), Nestin/Map2 (red/green), and Map2/TH (red/green). B, RT-PCR analysis of PIRX3, FOXA2, AADC, and HPRT (control) gene expression levels in NPCs and iDA neurons from four of the lines. C, GCase activity (percentage of control) was measured in NPCs from the iPSC lines indicated. Graph represents data from two independent experiments in quadruplicate. ${ }^{* * *} p=0.0003$ and $p=0.00022$. $\boldsymbol{D}$, Western blot analysis of GCase in NPCs, using the GCase-specific irreversible inhibitor MDW933. Tuj1 and $\beta$-actin were used as loading controls. $E$, Western blot analysis of TH levels in iDA neurons after $100 \mathrm{~d}$ in culture. A total of $10 \mu \mathrm{g}$ of protein was loaded per lane.

two subjects with GD1 alone had 15-fold and 10-fold \pm 0.09 higher levels of GlcSph than control iDA neurons. iDA neurons from the two subjects with GD1-PD showed 16.2 \pm 0.04 -fold and $36.2 \pm 0.18$ elevations in GlcSph, whereas in GD2 iDA neurons, GlcSph levels were $45.3 \pm 0.19$ times higher than control. The accumulation was specifically related to GCase deficiency, as the vast majority of sphingosine detected was GlcSph and not GalSph (Fig. 6B). Treatment with NCGC607 resulted in significantly reduced GlcSph levels in all five GD lines, indicating that the enhanced translocation of mutant GCase increased GlcSph hydrolysis (Fig. 6C).

GlcCer levels, evaluated by both mass spectrometry (Fig. $6 A, D)$ and immunostaining (Fig. $6 E$ ), were significantly elevated in aged GD1, GD1-PD, and GD2 iDA neurons. Treatment with NCGC607 resulted in a reduction of GlcCer levels, particularly in GD1-PD and GD2 iDA neurons (Fig. 6D). Staining of iDA neurons with an antibody against GlcCer confirmed the accu- mulation of GlcCer in the lysosomes of GD1, GD2, and GD1-PD iDA neurons, which was reduced after treatment with NCGC607 (Fig. 6E).

\section{$\alpha$-Syn accumulation in GD-PD iDA neurons is reversed} with NCGC607

The observation that reduced GCase leads to $\alpha$-syn accumulation in cultured neurons (Mazzulli et al., 2011) prompted us to study levels in our iDA neurons, kept in culture for $>150 \mathrm{~d}$. Staining for the iDA neuronal marker TH and for $\alpha$-syn (Fig. 7A) showed that $\alpha$-syn is present both in the neurite and cell body in GD iDA neurons, whereas in control neurons $\alpha$-syn was only observed in neurites. Moreover, while $\alpha$-syn protein levels were elevated in GD1-PD and GD2, but not GD1 iDA neurons (Fig. $7 B, C$ ), we did not observe significant differences in mRNA levels between GD and GD-PD iDA neurons (Fig. 7D). Next, we stained aged iDA neurons for Lamp2 and $\alpha$-syn and found colocalization of $\alpha$-syn 
A

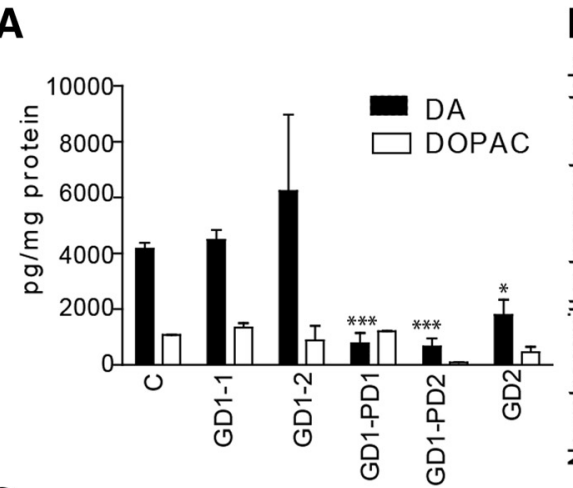

C

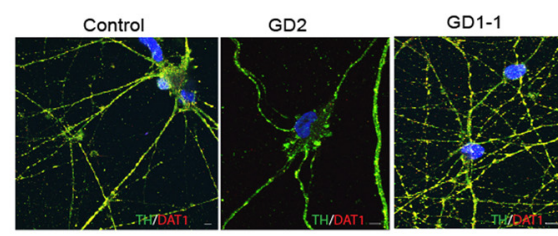

D
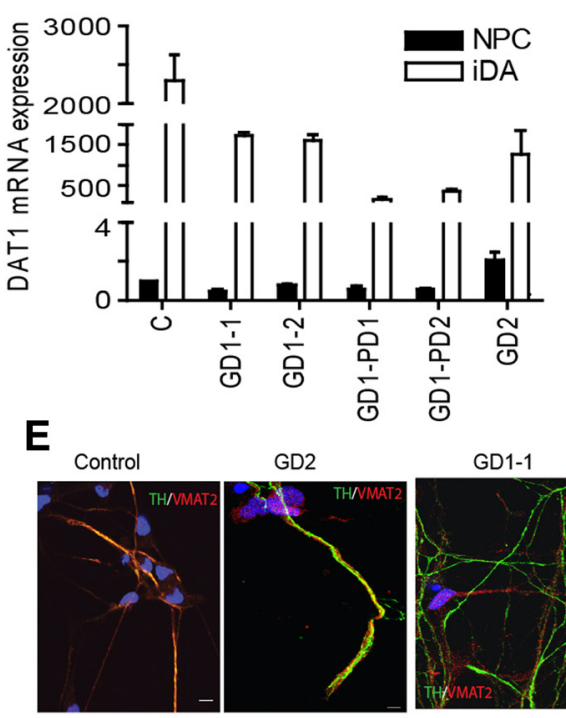
GD2

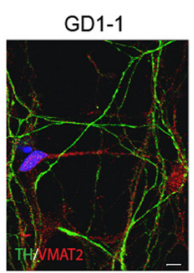

G

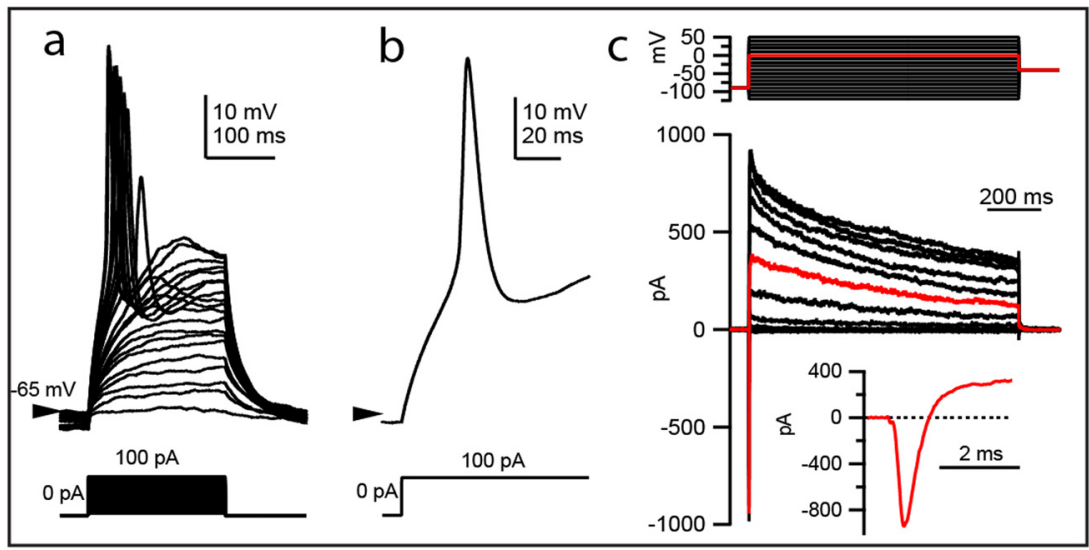

Figure 4. Dopamine release and uptake in iPSC-derived GD iDA neurons. $A, D A$ and 3,4-dihydroxyphenylacetic acid (DOPAC) were measured in iDA neurons. Data represent two independent experiments in triplicates: ${ }^{* * *} p=0.0038 ;{ }^{* * *} p=0.0016 ;{ }^{*} p=$ 0.043. B, Dopamine uptake in iDA neurons in the presence and absence of 10 and $20 \mu \mathrm{MGBR} 12909$, used as a specific DAT inhibitor. Graph represents data from three independent experiments in pentaplicates: ${ }^{*} p=0.043 ;{ }^{* * *} p=0.0042$. C, $\boldsymbol{E}$, Immunofluorescence analyses of GD1, GD2, or GD1-PD iDA neurons after $100 \mathrm{~d}$ in culture costained for TH (green), DAT1 (red) (C), or VMAT2 (red) $(\boldsymbol{E})$. Insets, Single-channel DAT1 or VMAT2. Scale bars, $5 \mu \mathrm{m}$. $\boldsymbol{D}, \boldsymbol{F}, \mathrm{RT}-\mathrm{PCR}$ analysis of DAT1 in NPCs and iDA neurons. Graphs represent two independent experiments. $\mathbf{G}$, Action potential firing in GD2 iDA neurons. $\mathbf{G} \boldsymbol{a}, \mathbf{G} \boldsymbol{b}$, Whole-cell recordings of spikes with Lamp2 in GD1-PD and GD2 iDA neurons indicating the presence of $\alpha$-syn in lysosomes. Treating aged iDA neurons with NCGC607 resulted in reduced $\alpha$-syn levels in GD1-PD and GD2 iDA neurons and reduced colocalization of $\alpha$-syn with Lamp2 (Fig. 7 E, F).

\section{Discussion}

It is increasingly clear that glucocerebrosidase plays a role in the pathogenesis of different forms of parkinsonism, as mutations in $G B A 1$ are a common genetic risk factor for PD and DLB, and both GBA1 homozygotes and heterozygotes are at risk (Sidransky et al., 2009; Nalls et al., 2013). Moreover, patients with idiopathic PD also appear to have decreased GCase levels. In this study, we generated and characterized iPSC-derived dopaminergic neurons from patients with GD and GD1-PD to further investigate this association. Although iPSC-iDA have been generated from subjects with GBA1 mutations previously (Schöndorf et al., 2014; Woodard et al., 2014; Sun et al., 2015; Fernandes et al., 2016), our study is unique, as we compared lines from subjects with GD with and without PD, including one set of siblings with genotype N370S/ N370S who were discordant for PD. This enabled us to better discriminate which aspects of the observed cellular phenotype were specifically associated with the development of parkinsonism. We also compared a line from a child with GD2 to those with GD1 and identified differences that could be related to the pathogenesis of neuronopathic GD. Furthermore, all lines were generated from patients who had undergone repeated medical evaluations by the investigators.

The six iPSC lines studied were differentiated first into NPCs and then iDA neurons. The GD1, GD1-PD, and GD2 samples had appropriately low GCase activity and GCase protein levels at different neurodevelopmental stages, which resulted in substrate accumulation, GlcCer and GlcSph.

It has been shown that dopaminergic neurons with mutant Parkin exhibit increased transcription of monoamine oxidases, increased DA release, and reduced DA uptake (Jiang et al., 2012). In contrast

\section{$\leftarrow$}

evoked by a range of current injections. Gc, Family of current traces evoked by voltage steps covering a range of potentials ( $5 \mathrm{mV}$ increments). Note the presence of transient outward current characteristic of potassium currents in native DA neurons. Red tracing represents current evoked by step to $0 \mathrm{mV}$. Inset, Fast inward $\mathrm{Na}^{+}$-mediated component. 
A

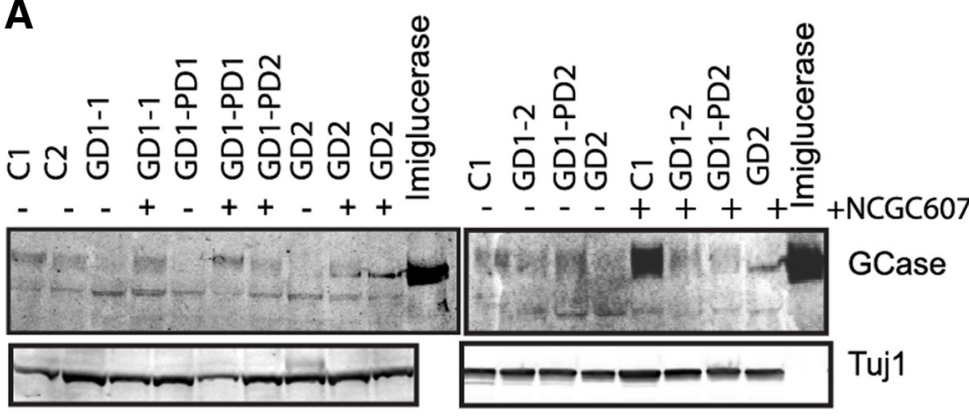

C

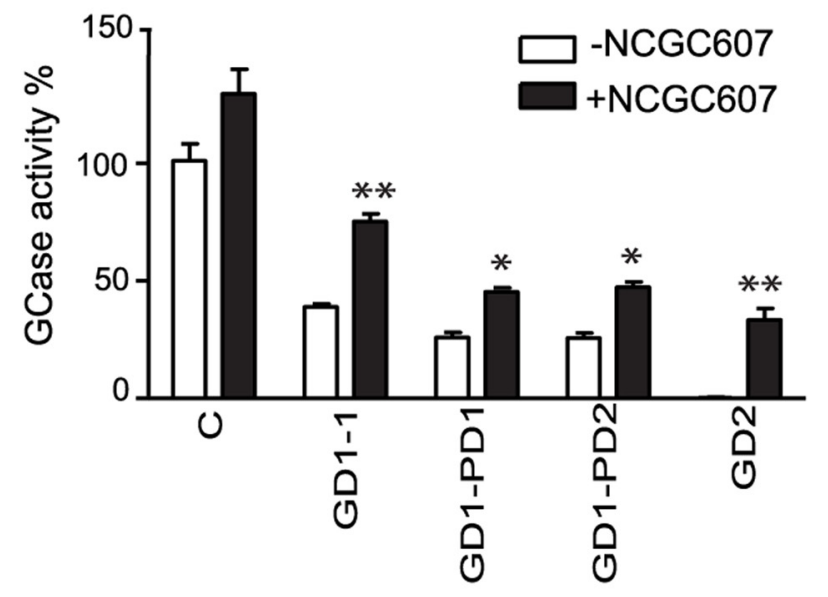

B

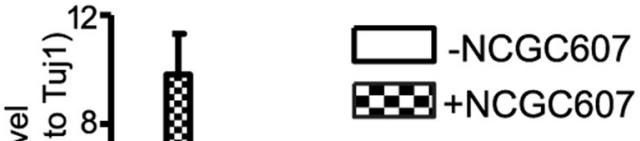

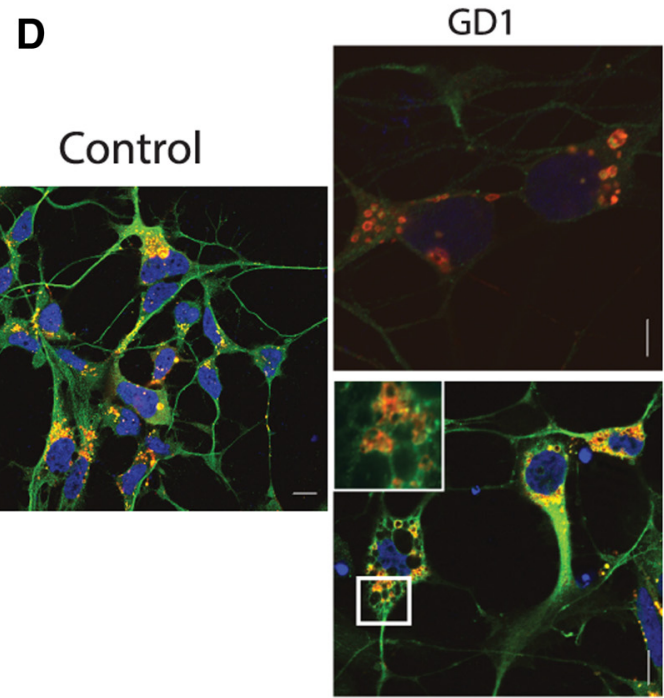
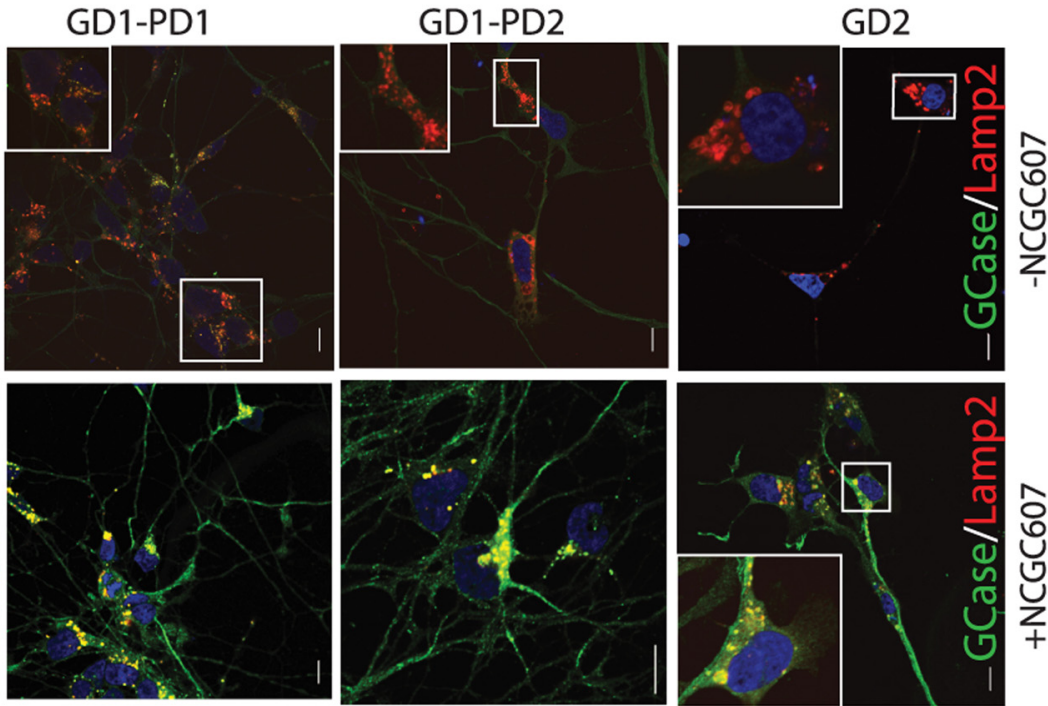

Figure 5. Small-molecule NCGC607 increases GCase activity and translocation to lysosomes. $A$, Western blot analysis of GCase in untreated and NCGC6070-treated iDA neurons using green fluorescent MDW933. Tuj1 was used as the loading control. Imiglucerase (recombinant GCase) was loaded as a positive control. B, Graph represents quantification of the blots in $\boldsymbol{A}$. C, GCase activity (percentage control) in iDA neurons in the presence and absence of NCGC607 (3 $\mu \mathrm{M})$. Graph represents data from three independent experiments in quadruplicate: ${ }^{* *} p=0.005 ;{ }^{*} p=0.02 ;{ }^{* *} p=$ 0.0025. D, Immunofluorescence study of iDA neurons before and after treatment with NCGC607 (3 $\mu \mathrm{m})$ for $21 \mathrm{~d}$. Cells were costained for GCase (green), Lamp2 (red), and DAPI (blue). Z-stack images were acquired using a Zeiss 510 confocal microscope $(63 \times$ magnification). Insets, Higher magnification of the areas outlined in the images. Scale bars, $5 \mu \mathrm{m}$. $\boldsymbol{E}$, The degree of colocalization reflected in Pearson's coefficient in areas of colocalization using Imaris software. Each data point represents the mean value of at least five cells per field.

dopaminergic neurons with mutation G2019S in LRRK2 show reduced DA release (Nguyen et al., 2011). In our study, GD1-PD iDA neurons appear to have reduced vesicular DA levels and DA uptake compared with control and GD1 neurons.
The plasma membrane proteins DAT and VMAT2 are essential for DA transmission. DAT rapidly removes DA from synapses, whereas VMAT2 transfers cytoplasmic DA into vesicles for storage and subsequent release. We observed reduced 

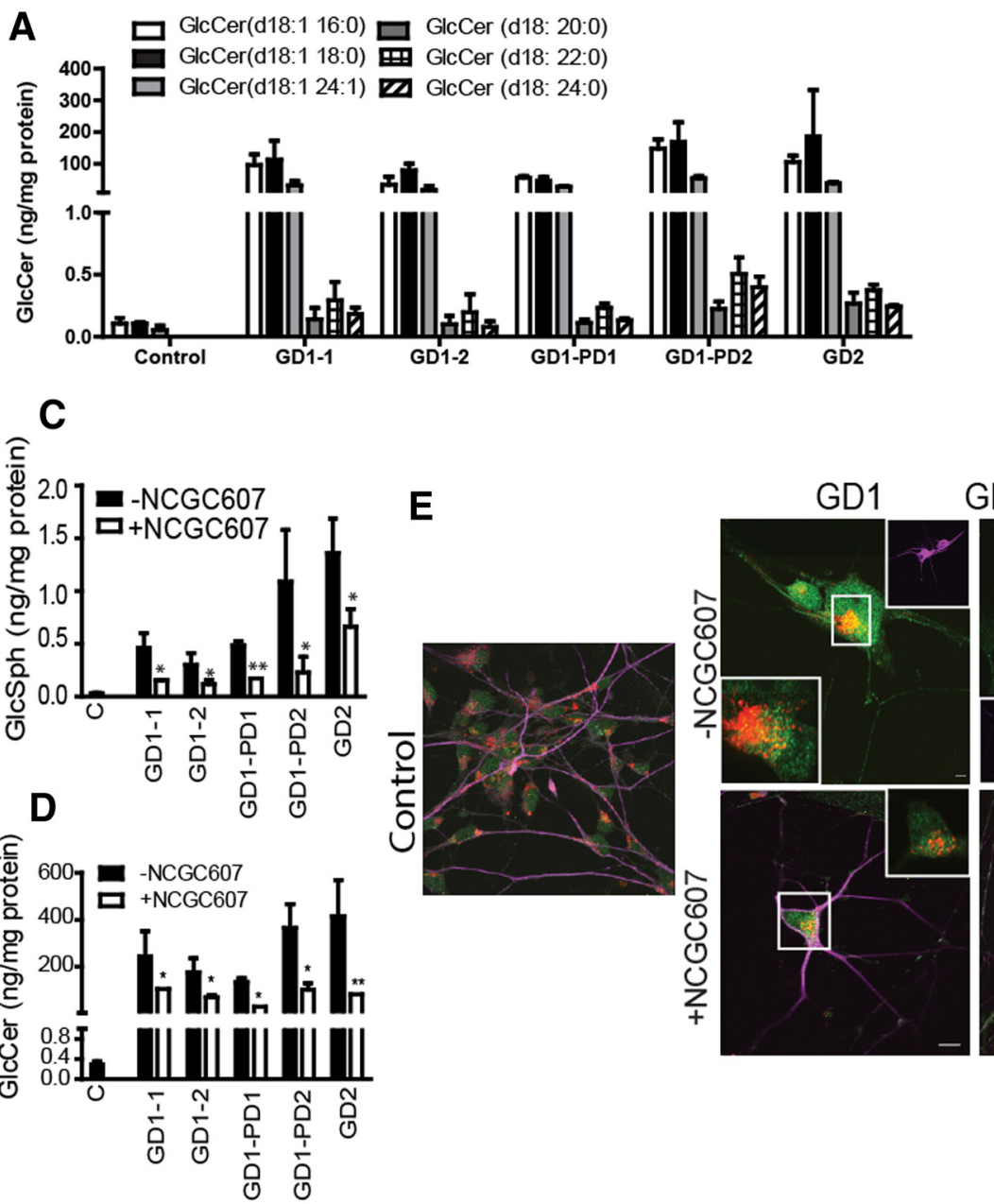

B

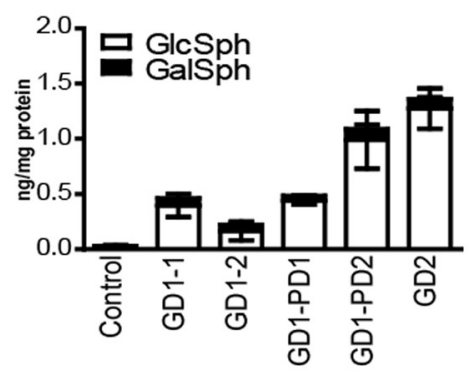

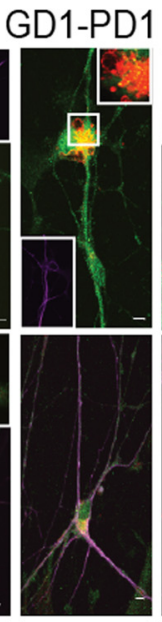
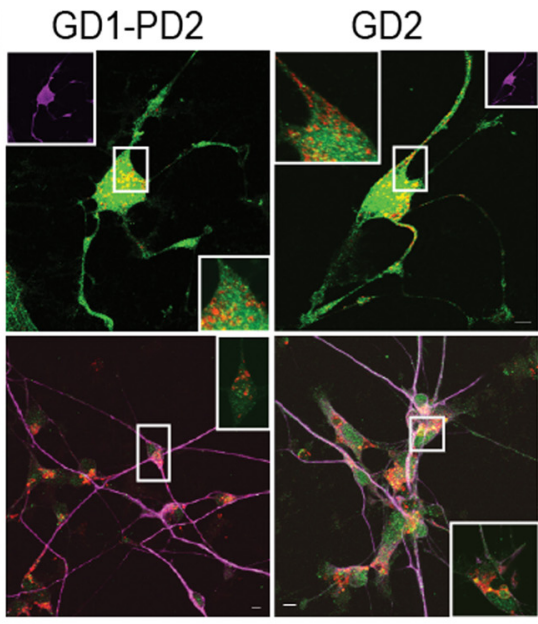

Figure 6. NGGC607 reduces GICSph and GIcCer accumulation in GD iDA neurons. $A$, Levels of different species of glucosylceramide in iDA neurons measured by mass spectrometry. $\boldsymbol{B}$, GICSph and GalSph measured in iDA neurons using HPLC/MS/MS. The portion from GalSph is shown in black. C, GlcSph levels. ${ }^{*} p=0.013, p=0.047, p=0.034$, and $p=0.031$ for GD1-1, GD1-2, GD1-PD2, and GD2, respectively. ${ }^{* *} p=0.0034$ for GD1-PD1. D, GlcCer levels. ${ }^{*} p=0.045, p=0.035, p=0.025$, and $p=0.02$ for GD1-1, GD1-2, GD1-PD1, and GD1-PD2, respectively, in iDA neurons after treatment with $3 \mu \mathrm{m} \mathrm{NCGC607} \mathrm{for} 21 \mathrm{~d}$. ${ }^{* *} p=0.0016$ for GD2 in iDA neurons after treatment with $3 \mu \mathrm{m}$ NCGC607 for $21 \mathrm{~d}$. Graph represents the mean of two independent experiments $(n=6)$. $\boldsymbol{E}$, Immunofluorescence staining with antibodies to GlcCer (green), Lamp2 (red), and Map2 (pink) in iDA neurons in the presence and absence of NCGC607. Nuclei are counterstained with DAPI. Insets, Higher magnification of the areas outlined in the images. Insets stained in pink, Single-channel views of Map2. Scale bars, $5 \mu \mathrm{m}$.

DAT and VMAT2 expression levels in GD1-PD iDA neurons, which likely contributes to reduced DA uptake in these cells.

Because there is evidence of a reciprocal relationship between GCase and $\alpha$-syn, one potential strategy for the treatment of PD involves enhancement of GCase. Although enzyme replacement therapy successfully treats the nonneurological symptoms of GD, the treatment is not effective for neuronopathic GD (Altarescu et al., 2001; Weinreb et al., 2002) as it does not cross the blood-brain barrier, and there are reports of patients with GD who developed PD while on enzyme replacement therapy (Goker-Alpan et al., 2008). Thus, chemical chaperone therapy has been proposed as an alternative strategy for both patients with GD and PD. In this study, we introduce a new noninhibitory small molecule identified through high throughput screening and structure-activity relationship studies, NCGC607, which is able to restore GCase activity and reduce substrate storage in iMacs and iDA neurons from patients with GD1, GD1-PD, and GD2. Unlike the iminosugar molecules isofagomine and $N$-butyl-deoxynorjirimycin, commonly proposed as GCase chaperones, NCGC607 does not inhibit GCase specific activity in vitro or in cells (Marugan et al., 2012). $\alpha$-syn aggregation is a pathological hallmark of PD and associated synucleinopathies. Neuropathologic studies of brains from subjects with PD with GBA1 mutations reveal extensive $\alpha$-syn pathology and inclusions, as well as the presence of GCase in LBs (Goker-Alpan et al., 2010). Moreover, patients with sporadic PD without GBA1 mutations also have reduced GCase activity that is associated with increased $\alpha$-syn levels (Murphy et al., 2014). Although the exact mechanism is still unknown, it appears that GBA1 mutations play a pivotal role, reflected in enhanced aggregation of $\alpha$-syn. One theory is that reduced GCase activity contributes to lysosomal dysfunction, which may also lead to $\alpha$-syn accumulation (Mazzulli et al., 2011). Using iPSC-derived DA neurons, we show that GD1-PD and GD2 iDA neurons indeed do exhibit elevated levels of $\alpha$-syn. This was not seen in iDA neurons from patients with GD without $\mathrm{PD}$, which may correspond to the clinical observation that only a minority of subjects with GBA1 mutations go on to develop parkinsonism.

The study also enabled us to compare the cellular phenotype of iDA neurons from patients from GD1 and GD2. As expected, those from GD2 have greater GCase deficiency and more glycolipid storage, both of which improved considerably 
A

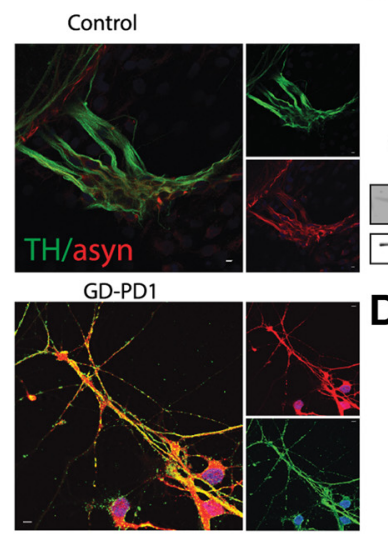

GD1

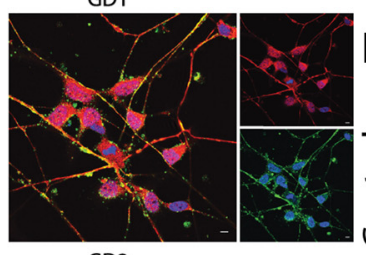

GD2

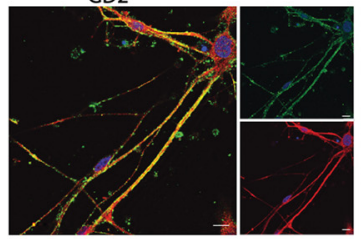

GD-PD2

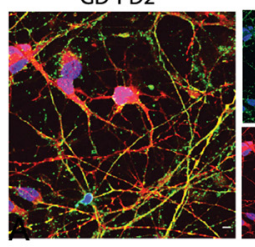

B

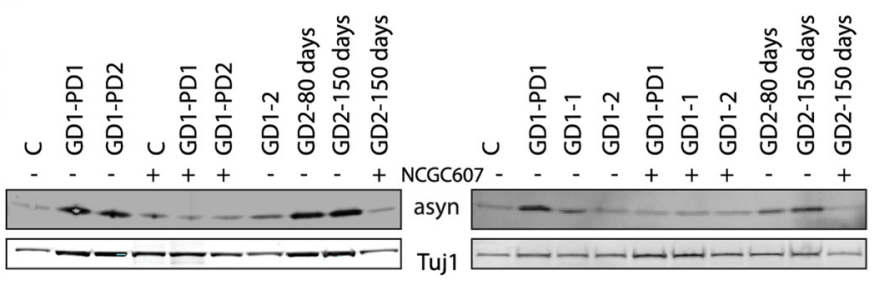

D

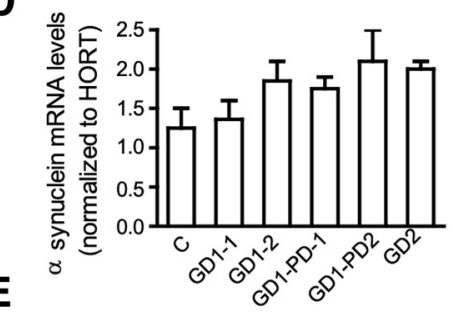

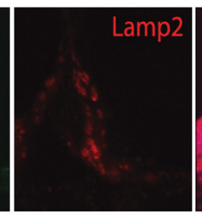
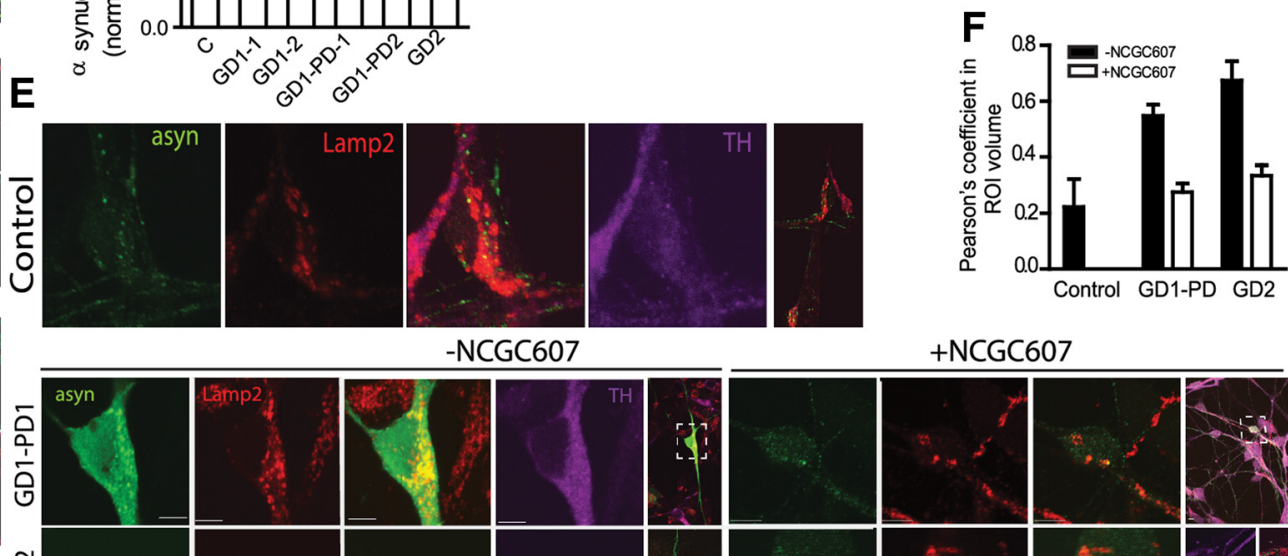

NCGC607
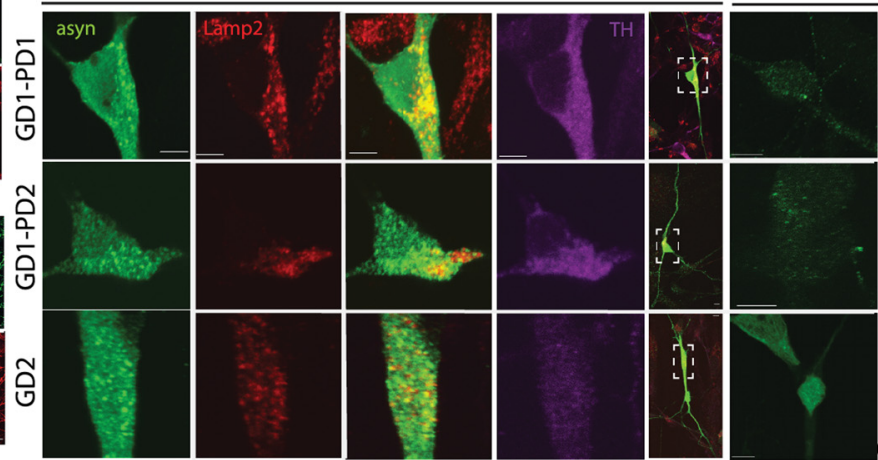

+ NCGC607

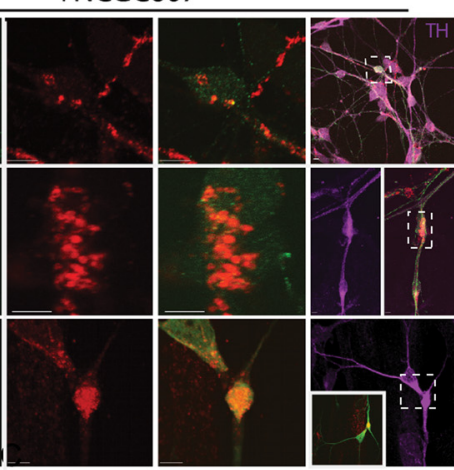

Figure 7. $\alpha$-Syn levels in iDA neurons are reduced after NCGC607 treatment. A, Immunofluorescence analysis of iDA neurons stained with antibody to TH (green) and $\alpha$-syn (red). Scale bars, $5 \mu \mathrm{m}$. B. Western blot analysis of $\alpha$-syn levels in iDA neurons after $150 \mathrm{~d}$ in culture and treated with $3 \mu \mathrm{m} \mathrm{NCGC607} \mathrm{for} 21 \mathrm{~d}$. Tuj 1 was used as the loading control. C, Quantification of the $\alpha$-syn levels in iDA neurons in the presence and absence of NCGC607. Statistically significant differences between the untreated and treated cells: ${ }^{* * *} p=0.00017 ; p=0.0005$;

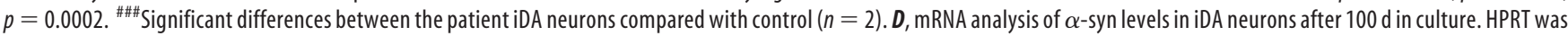
used as internal control. Graph represents the mean of two independent experiments. $\boldsymbol{E}$, iDA neurons were costained for $\alpha$-syn (green), Lamp2 (red), and TH (purple) in the absence (left) and presence (right) of NCGC607 (3 $\mu \mathrm{m}, 21 \mathrm{~d}$ ). Insets, Higher magnification of the areas outlined in the images. Z-stack images were acquired using a Zeiss 510 confocal microscope ( $63 \times$ magnification). Scale bars, $5 \mu \mathrm{m}$. $\boldsymbol{F}$, Colocalization between Lamp2 and $\alpha$-syn was measured for 50 individual cells and Pearson's coefficient quantified in R0I volume, using Imaris software.

with NCGC607 treatment. The GD2 line had genotype L444P/ IVS2 $+1 \mathrm{G}>\mathrm{T}$, indicating that the small-molecule chaperoned mutations other than N370S. Remarkably, GD2 iDAs differ from GD1 in that they accumulated $\alpha$-syn at levels similar to what is seen in our GD1-PD lines. This was also reported in brain samples from subjects with GD2 (Mazzulli et al., 2011) and could be related to the profound GCase deficiency in these babies. Although parkinsonism is not associated with GD2, early mortality is universal in these patients.

We then show that our new noninhibitory chaperone NCGC607 successfully decreases the elevated $\alpha$-syn levels in GD1-PD and GD2 iDA neurons. Although in control iDA neurons there is minimal $\alpha$-syn in lysosomes, enhanced colocalization between $\alpha$-syn and the lysosomal marker Lamp2 is observed in GD1-PD and GD2 iDA neurons. Treatment with the small-molecule NCGC607 led to reduced colocalization, suggesting that this is a direct result of increased lysosomal GCase. Our findings support the theory that lysosomal dysfunction associated with diminished GCase activity might contribute to the accumulation of $\alpha$-syn. The study provides further proof of principle that increasing GCase activity is a viable strategy for reducing $\alpha$-syn levels.

A unique feature of this work was its focus on patients with GD with and without parkinsonism. To our knowledge, this is the first report of the generation and characterization of iPSCderived iDA neurons from patients sharing the two phenotypes compared with subjects with GD alone. Our ability to pharmacologically correct the phenotype in all three lines provides validation that the findings observed were not spurious phenotypes. Furthermore, this is the first introduction of NCGC607, demonstrating its ability to reverse multiple aspects of the cellular and biochemical phenotype in an appropriate cell-based assay. Future studies should address the ability of NCGC607 to modulate $\alpha$-syn in lines generated from subjects with other homozygous and heterozygous GBA1 ge- 
notypes, idiopathic PD, as well as synucleinopathies of other etiologies.

In conclusion, our study shows that GD1, GD1-PD, and GD2 iDA neurons have reduced GCase activity, decreased intracellular DA and DA uptake, as well as elevated levels of GlcSph and GlcCer. Lines from subjects with GD1-PD and GD2 also have increased $\alpha$-syn. Treatment with the new noninhibitory small-molecule NCGC607 successfully restored GCase activity and protein levels and reduced GlcSph and GlcCer levels in both iMacs and iDA neurons, indicating that this chemical class has potential for further development as a therapy for GD, including neuronopathic forms. In addition, NCGC607 significantly reduced $\alpha$-syn levels in iDA neurons from subjects with GD1-PD, demonstrating how modulating lysosomal GCase impacts $\alpha$-syn accumulation. Our results suggest that compounds targeting GCase may have utility for the treatment of Parkinson disease.

\section{References}

Aflaki E, Stubblefield BK, Maniwang E, Lopez G, Moaven N, Goldin E, Marugan J, Patnaik S, Dutra A, Southall N, Zheng W, Tayebi N, Sidransky E (2014) Macrophage models of Gaucher disease for evaluating disease pathogenesis and candidate drugs. Sci Transl Med 6:240ra273. CrossRef Medline

Altarescu G, Hill S, Wiggs E, Jeffries N, Kreps C, Parker CC, Brady RO, Barton NW, Schiffmann R (2001) The efficacy of enzyme replacement therapy in patients with chronic neuronopathic Gaucher's disease. J Pediatr 138: 539-547. CrossRef Medline

Fernandes HJ, Hartfield EM, Christian HC, Emmanoulidou E, Zheng Y, Booth H, Bogetofte H, Lang C, Ryan BJ, Sardi SP, Badger J, Vowles J, Evetts S, Tofaris GK, Vekrellis K, Talbot K, Hu MT, James W, Cowley SA, Wade-Martins R (2016) ER stress and autophagic perturbations lead to elevated extracellular alpha-synuclein in GBA-N370S Parkinson's iPSCderived dopamine neurons. Stem Cell Rep 6:342-356. CrossRef Medline

Gegg ME, Burke D, Heales SJ, Cooper JM, Hardy J, Wood NW, Schapira AH (2012) Glucocerebrosidase deficiency in substantia nigra of Parkinson disease brains. Ann Neurol 72:455-463. CrossRef Medline

Goker-Alpan O, Schiffmann R, LaMarca ME, Nussbaum RL, McInerney-Leo A, Sidransky E (2004) Parkinsonism among Gaucher disease carriers. J Med Genet 41:937-940. CrossRef Medline

Goker-Alpan O, Lopez G, Vithayathil J, Davis J, Hallett M, Sidransky E (2008) The spectrum of parkinsonian manifestations associated with glucocerebrosidase mutations. Arch Neurol 65:1353-1357. CrossRef Medline

Goker-Alpan O, Stubblefield BK, Giasson BI, Sidransky E (2010) Glucocerebrosidase is present in alpha-synuclein inclusions in Lewy body disorders. Acta Neuropathol 120:641-649. CrossRef Medline

Goker-Alpan O, Masdeu JC, Kohn PD, Ianni A, Lopez G, Groden C, Chapman MC, Cropp B, Eisenberg DP, Maniwang ED, Davis J, Wiggs E, Sidransky E, Berman KF (2012) The neurobiology of glucocerebrosidase-associated parkinsonism: a positron emission tomography study of dopamine synthesis and regional cerebral blood flow. Brain 135:24402448. CrossRef Medline

Goldin E, Zheng W, Motabar O, Southall N, Choi JH, Marugan J, Austin CP, Sidransky E (2012) High throughput screening for small molecule therapy for Gaucher disease using patient tissue as the source of mutant glucocerebrosidase. PLoS One 7:e29861. CrossRef Medline

Goldstein DS, Sullivan P, Cooney A, Jinsmaa Y, Sullivan R, Gross DJ, Holmes C, Kopin IJ, Sharabi Y (2012) Vesicular uptake blockade generates the toxic dopamine metabolite 3,4-dihydroxyphenylacetaldehyde in PC12 cells: relevance to the pathogenesis of Parkinson's disease. J Neurochem 123:932-943. CrossRef Medline

Hartfield EM, Yamasaki-Mann M, Ribeiro Fernandes HJ, Vowles J, James WS, Cowley SA, Wade-Martins R (2014) Physiological characterisation of human iPS-derived dopaminergic neurons. PLoS One 9:e87388. CrossRef Medline

Jiang H, Ren Y, Yuen EY, Zhong P, Ghaedi M, Hu Z, Azabdaftari G, Nakaso K, Yan Z, Feng J (2012) Parkin controls dopamine utilization in human midbrain dopaminergic neurons derived from induced pluripotent stem cells. Nat Commun 3:668. CrossRef Medline
Kono S, Ouchi Y, Terada T, Ida H, Suzuki M, Miyajima H (2010) Functional brain imaging in glucocerebrosidase mutation carriers with and without parkinsonism. Mov Disord 25:1823-1829. CrossRef Medline

Kriks S, Shim JW, Piao J, Ganat YM, Wakeman DR, Xie Z, Carrillo-Reid L, Auyeung G, Antonacci C, Buch A, Yang L, Beal MF, Surmeier DJ, Kordower JH, Tabar V, Studer L (2011) Dopamine neurons derived from human ES cells efficiently engraft in animal models of Parkinson's disease. Nature 480:547-551. CrossRef Medline

Marugan JJ, Huang W, Motabar O, Zheng W, Xiao J, Patnaik S, Southall N, Westbroek W, Lea WA, Simeonov A, Goldin E, Debernardi MA, Sidransky E (2012) Non-iminosugar glucocerebrosidase small molecule chaperones. Med Chem Commun 3:56-60. CrossRef Medline

Mazzulli JR, Xu YH, Sun Y, Knight AL, McLean PJ, Caldwell GA, Sidransky E, Grabowski GA, Krainc D (2011) Gaucher disease glucocerebrosidase and alpha-synuclein form a bidirectional pathogenic loop in synucleinopathies. Cell 146:37-52. CrossRef Medline

Murphy KE, Gysbers AM, Abbott SK, Tayebi N, Kim WS, Sidransky E, Cooper A, Garner B, Halliday GM (2014) Reduced glucocerebrosidase is associated with increased alpha-synuclein in sporadic Parkinson's disease. Brain 137:834-848. CrossRef Medline

Nalls MA, Duran R, Lopez G, Kurzawa-Akanbi M, McKeith IG, Chinnery PF, Morris CM, Theuns J, Crosiers D, Cras P, Engelborghs S, De Deyn PP, Van Broeckhoven C, Mann DM, Snowden J, Pickering-Brown S, Halliwell N, Davidson Y, Gibbons L, Harris J, et al. (2013) A multicenter study of glucocerebrosidase mutations in dementia with Lewy bodies. JAMA Neurol 70:727-735. CrossRef Medline

Nguyen HN, Byers B, Cord B, Shcheglovitov A, Byrne J, Gujar P, Kee K, Schüle B, Dolmetsch RE, Langston W, Palmer TD, Pera RR (2011) LRRK2 mutant iPSC-derived DA neurons demonstrate increased susceptibility to oxidative stress. Cell Stem Cell 8:267-280. CrossRef Medline

Nirenberg MJ, Chan J, Liu Y, Edwards RH, Pickel VM (1996) Ultrastructural localization of the vesicular monoamine transporter-2 in midbrain dopaminergic neurons: potential sites for somatodendritic storage and release of dopamine. J Neurosci 16:4135-4145. Medline

Pevny LH, Sockanathan S, Placzek M, Lovell-Badge R (1998) A role for SOX1 in neural determination. Development 125:1967-1978. Medline

Richter F, Fleming SM, Watson M, Lemesre V, Pellegrino L, Ranes B, Zhu C, Mortazavi F, Mulligan CK, Sioshansi PC, Hean S, De La Rosa K, Khanna R, Flanagan J, Lockhart DJ, Wustman BA, Clark SW, Chesselet MF (2014) A GCase chaperone improves motor function in a mouse model of synucleinopathy. Neurotherapeutics 11:840-856. CrossRef Medline

Saunders-Pullman R, Hagenah J, Dhawan V, Stanley K, Pastores G, Sathe S, Tagliati M, Condefer K, Palmese C, Brüggemann N, Klein C, Roe A, Kornreich R, Ozelius L, Bressman S (2010) Gaucher disease ascertained through a Parkinson's center: imaging and clinical characterization. Mov Disord 25:1364-1372. CrossRef Medline

Sawkar AR, Schmitz M, Zimmer KP, Reczek D, Edmunds T, Balch WE, Kelly JW (2006) Chemical chaperones and permissive temperatures alter localization of Gaucher disease associated glucocerebrosidase variants. ACS Chem Biol 1:235-251. CrossRef Medline

Schapira AH (2015) Glucocerebrosidase and Parkinson disease: recent advances. Mol Cell Neurosci 66:37-42. CrossRef Medline

Schöndorf DC, Aureli M, McAllister FE, Hindley CJ, Mayer F, Schmid B, Sardi SP, Valsecchi M, Hoffmann S, Schwarz LK, Hedrich U, Berg D, Shihabuddin LS, Hu J, Pruszak J, Gygi SP, Sonnino S, Gasser T, Deleidi M (2014) iPSC-derived neurons from GBA1-associated Parkinson's disease patients show autophagic defects and impaired calcium homeostasis. Nat Commun 5:4028. CrossRef Medline

Sidransky E, Nalls MA, Aasly JO, Aharon-Peretz J, Annesi G, Barbosa ER, Bar-Shira A, Berg D, Bras J, Brice A, Chen CM, Clark LN, Condroyer C, De Marco EV, Dürr A, Eblan MJ, Fahn S, Farrer MJ, Fung HC, Gan-Or Z, et al. (2009) Multicenter analysis of glucocerebrosidase mutations in Parkinson's disease. N Engl J Med 361:1651-1661. CrossRef Medline

Siebert M, Sidransky E, Westbroek W (2014) Glucocerebrosidase is shaking up the synucleinopathies. Brain 137:1304-1322. CrossRef Medline

Sun Y, Florer J, Mayhew CN, Jia Z, Zhao Z, Xu K, Ran H, Liou B, Zhang W, Setchell KD, Gu J, Grabowski GA (2015) Properties of neurons derived from induced pluripotent stem cells of Gaucher disease Type 2 patient fibroblasts: potential role in neuropathology. PLoS One 10:e0118771. CrossRef Medline

Tayebi N, Walker J, Stubblefield B, Orvisky E, LaMarca ME, Wong K, Rosenbaum H, Schiffmann R, Bembi B, Sidransky E (2003) Gaucher disease 
with parkinsonian manifestations: does glucocerebrosidase deficiency contribute to a vulnerability to parkinsonism? Mol Genet Metab 79:104109. CrossRef Medline

Weinreb NJ, Charrow J, Andersson HC, Kaplan P, Kolodny EH, Mistry P, Pastores G, Rosenbloom BE, Scott CR, Wappner RS, Zimran A (2002) Effectiveness of enzyme replacement therapy in 1028 patients with Type 1 Gaucher disease after 2 to 5 years of treatment: a report from the Gaucher Registry. Am J Med 113:112-119. CrossRef Medline

Weiss K, Gonzalez AN, Lopez G, Pedoeim L, Groden C, Sidransky E (2015) The clinical management of Type 2 Gaucher disease. Mol Genet Metab 114:110-122. CrossRef Medline
Witte MD, Kallemeijn WW, Aten J, Li KY, Strijland A, Donker-Koopman WE, van den Nieuwendijk AM, Bleijlevens B, Kramer G, Florea BI, Hooibrink B, Hollak CE, Ottenhoff R, Boot RG, van der Marel GA, Overkleeft HS, Aerts JM (2010) Ultrasensitive in situ visualization of active glucocerebrosidase molecules. Nat Chem Biol 6:907-913 CrossRef Medline

Woodard CM, Campos BA, Kuo SH, Nirenberg MJ, Nestor MW, Zimmer M, Mosharov EV, Sulzer D, Zhou H, Paull D, Clark L, Schadt EE, Sardi SP, Rubin L, Eggan K, Brock M, Lipnick S, Rao M, Chang S, Li A, et al. (2014) iPSC-derived dopamine neurons reveal differences between monozygotic twins discordant for Parkinson's disease. Cell Rep 9:1173-1182. CrossRef Medline 